\title{
Phenotype characterization of human melanoma cells resistant to dabrafenib
}

\author{
FABIOLA GILDA CORDARO ${ }^{1}$, ANNA LISA DE PRESBITERIS ${ }^{1}$, ROSA CAMERLINGO ${ }^{2}$, NICOLA MOZZILLO $^{2}$, \\ GIUSEPPE PIROZZI ${ }^{2}$, ERNESTA CAVALCANTI ${ }^{2}$, ANTONELLA MANCA ${ }^{3}$, GIUSEPPE PALMIERI $^{3}$, \\ ANTONIO COSSU ${ }^{4}$, GENNARO CILIBERTO ${ }^{2}$, PAOLO A. ASCIERTO ${ }^{2}$, SALVATORE TRAVALI $^{5}$, \\ EDUARDO J. PATRIARCA ${ }^{1}$ and EMILIA CAPUTO ${ }^{1}$ \\ ${ }^{1}$ Institute of Genetics and Biophysics (IGB), A. Buzzati-Traverso, CNR, I-80131 Naples; \\ ${ }^{2}$ Istituto Nazionale Tumori Fondazione G. Pascale, I-80131 Naples; ${ }^{3}$ Unit of Cancer Genetics, \\ Institute of Biomolecular Chemistry, CNR, I-07100 Sassari; ${ }^{4}$ Unit of Pathology, \\ Hospital-University Health Unit (AOU), I-07100 Sassari; ${ }^{5}$ Department of Biomedical and \\ Biotechnological Sciences-BIOMETEC, University of Catania, I-95100 Catania, Italy
}

Received August 29, 2016; Accepted March 10, 2017

DOI: $10.3892 /$ or.2017.5963

\begin{abstract}
In the present study, the phenotype of melanoma cells resistant to dabrafenib (a B-RAF inhibitor) was investigated, to shed more light on melanoma resistance to B-RAF inhibition. Melanoma cells resistant to dabrafenib were generated using 3 different cell lines, A375, 397 and 624.38, all carrying $\mathrm{B}-\mathrm{RAF}^{\mathrm{V} 600 \mathrm{E}}$, and they were characterized by cytofluorometric analysis, Ion Torrent technology, immunofluorescence and biochemistry. All dabrafenib-resistant cells showed, in addition to a re-activation of MAPK signaling, morphological changes compared to their sensitive counterparts, accompanied by an increase in CD90 (mesenchymal marker) expression and a decrease in E-cadherin (epithelial marker) expression, suggesting an epithelial-to-mesenchymal-like phenotypic transition. However, melanoma cells with TGF- $\beta 1$-induced epithelial-to-mesenchymal transition (EMT) were more sensitive to dabrafenib treatment compared to the sensitivity noted in the non-TGF- $\beta 1$-induced EMT melanoma cells, suggesting that TGF- $\beta 1$-induced EMT was not associated with dabrafenib resistance. Although dabrafenib-resistant cells exhibited increased cell motility and E-cadherin/vimentin
\end{abstract}

Correspondence to: Dr Emilia Caputo, Institute of Genetics and Biophysics (IGB), A. Buzzati-Traverso, CNR, 111 Via Pietro Castellino, I-80131 Naples, Italy

E-mail: emilia.caputo@igb.cnr.it

Abbreviations: MAPK, mitogen activated protein kinases; B-RAFi, B-RAF inhibitors; MEKi, MEK inhibitors; PI, propidium iodide; EMT, epithelial-to-mesenchymal transition; MM, metastatic melanoma

Key words: melanoma, targeted therapy, melanoma drug resistance, B-RAF inhibitor, MAPK signaling reorganization, as expected in EMT, all of them showed unvaried E-cadherin mRNA and unchanged Snail protein levels, while Twist1 protein expression was decreased with the exception of A375 dabrafenib-resistant melanoma cells, where it was unaffected. These findings suggest a distinct active EMT-like process adopted by melanoma cells under drug exposure. Furthermore, dabrafenib-resistant cells exhibited stem cell-like features, with Oct 4 translocation from the cytoplasm to peri-nuclear sites and nuclei, and increased CD20 expression. In conclusion, our data, in addition to confirming that resistance to dabrafenib is dependent on re-activation of MAPK signaling, suggest that this resistance is linked to a distinct active EMT-like process as well as stem-cell features adopted by melanoma cells.

\section{Introduction}

Melanoma is the most lethal form of skin cancer and represents more than $75 \%$ of skin cancer-related deaths (1). Metastatic melanoma (MM) is poorly responsive to treatment based on conventional chemotherapy, resulting in a 5-year survival rate of only $15 \%$ (2). Molecular alterations associated with sun exposure (3) or DNA methylation (4) have been identified as linked to melanoma development. In particular, gene mutations in the RAS/RAF/MEK/ERK or mitogen-activated protein kinase (MAPK) pathway have been detected (5) and have provided new targets for therapy.

Therapy targeting the mitogen-activated protein kinase (MAPK) pathway, with small molecules that inhibit the signal transmission between BRAF and MEK, have been effective in the clinical outcomes of the disease. Selective inhibitors of oncogenically activated BRAF, vemurafenib and dabrafenib, have demonstrated major tumor responses in $\sim 50 \%$ of patients (6).

Vemurafenib achieved more than a two-third decrease in the risk for both death and disease progression as compared with dacarbazine. However, the majority of patients exhibit 
drug resistance after 6-8 months due to several resistance mechanisms (7). Although these mechanisms are only partially understood, MAPK reactivation seems to be primarily responsible for acquired resistance, via B-RAF copy number gains, aberrant B-RAF splicing, mutations in N-RAS or MEK1/2 and upregulation of receptor tyrosine kinases (8-11).

MM treatment with a combination of B-RAF and MEK inhibitors (dabrafenib plus trametinib, and vemurafenib plus cobimetinib, respectively) have been used as a strategy to overcome drug resistance development (12). Approximately half of melanoma patients treated with a combination of dabrafenib/ trametinib exhibit drug resistance at 11-12 months (median progression-free survival, 11 months; median duration of response, 12.9 months) (13), although resistance remains a hurdle to achieve better patient outcomes.

Recently, we demonstrated that the B-RAF/MEK/AurkA inhibitor triple-combination treatment was more effective for inhibiting melanoma cell growth, using a 3D-human melanoma model (14). However, single or combined treatment was effective only on polygonal-shaped melanoma cells present at the epidermal/dermal junction site, while spindle-shaped melanoma cells, detected in the dermal stratum, continued to be alive and to proliferate.

In the present study, we characterized the phenotype of melanoma cells resistant to dabrafenib. To this end, we selected in vitro melanoma cells resistant to dabrafenib [B-RAF inhibitor (B-RAFi)] from 3 different dabrafenib-sensitive melanoma cell lines (A375, 397 and 624.38) and we performed a comparative phenotype study between dabrafenib-resistant and -sensitive melanoma cells, under drug selective pressure, since the resistance to $\mathrm{B}-\mathrm{RAF}^{\mathrm{V} 600 \mathrm{E}}$ inhibition in melanoma is reversible and adaptive (15).

\section{Materials and methods}

Cell culture and reagents. A375, 624.38 and 397 melanoma cell lines, kindly provided by Dr F. M. Marincola and Dr M. Bettinotti (NIH, Bethesda, MD, USA), were used. All cell lines were cultured in RPMI-1640 medium, supplemented with $3 \mathrm{mM}$ L-glutamine (both from Invitrogen-Gibco, Paisley, UK), $2 \%$ penicillin/streptomycin and $10 \%$ fetal bovine serum (FBS). The cell cultures were incubated at $37^{\circ} \mathrm{C}$ in a humidified $5 \% \mathrm{CO}_{2}$ atmosphere. Dabrafenib and trametinib were purchased from Selleck (Munich, Germany).

Colony formation assay. A375 (50 cells $\left./ \mathrm{cm}^{2}\right), 624.38$ $\left(50\right.$ cells $\left./ \mathrm{cm}^{2}\right)$ and $397\left(250\right.$ cells $\left./ \mathrm{cm}^{2}\right)$ melanoma cells were plated in 24-multi-wells, previously coated with $1 \%$ gelatin, with complete medium containing dabrafenib $(30 \mathrm{nM})$ or not containing the drug. The medium was replenished every 2 days. After 7 days, the cells were fixed in $4 \%$ paraformaldehyde (PFA) and stained with $0.15 \%$ crystal violet. Plates were imaged by scanner and colonies were imaged on a Leica DMI6000 inverted microscope (Leica, Mannheim, Germany). The observed number of colonies was determined from 5 independent areas using ImageJ software (http://rsbweb.nih.gov/ij/).

Selection and expansion of melanoma cell lines resistant to $B-R A F$ inhibitors. Dabrafenib-resistant melanoma cells were selected by growing all 3 melanoma cell lines in medium containing increased concentrations of dabrafenib for 4 weeks. A375 and 397 melanoma cell lines resistant to $30 \mathrm{nM}$ dabrafenib were selected, while for the 624.38 cell line melanoma cells resistant to $100 \mathrm{nM}$ dabrafenib were selected.

CyQUANT assay. Cells (1,500/well) were seeded in triplicate in 96-well plates. After $24 \mathrm{~h}$, the cells were treated with different drug concentrations. The monitoring of the number of cells following 6 days of drug treatment was performed using CyQUANT cell proliferation assay kit, according to the manufacturer's procedure (Invitrogen, Paisley, UK). Dose-response data were analyzed by GraphPad Prism version 5.00 for Windows (GraphPad Software) to determine the $\mathrm{IC}_{50}$ values.

Cell cycle analysis. Cells were harvested in phosphate-buffered saline (PBS) containing $2 \mathrm{mM}$ EDTA, washed once with PBS and fixed in ethanol at $96^{\circ} \mathrm{C}$. After washing in PBS, $1 \times 10^{6}$ cells were incubated with $5 \mu \mathrm{g} / \mathrm{ml}$ propidium iodide (PI) (Sigma Chemical Co., St. Louis, MO, USA) plus $25 \mu \mathrm{l}$ RNase $(1 \mathrm{mg} / \mathrm{ml})$, overnight at $4^{\circ} \mathrm{C}$ in the dark. Stained nuclei were analyzed using FACSAria II (Becton-Dickinson, Franklin Lakes, NJ, USA). Data were analyzed using a ModFit III (Verity, Topsham, ME, USA) cell cycle analysis programme.

Next generation sequencing (NGS) on the Ion Torrent ${ }^{\mathrm{TM}}$ platform. Genomic DNA was isolated from melanoma cell lines by standard methods and quantified using the Qubit $^{\circledR}$ fluorometer (Life Technologies, Gent, Belgium). For library construction, DNA (10 ng) was amplified using the Ion Torrent AmpliSeq Hotspot V2/CHPv2 Cancer Panel (Life Technologies-ThermoFisher Scientific, Waltham, MA, USA). An amplicon library was generated for sequencing $\sim 2,800$ mutations in the 50 most common oncogenes and tumorsuppressor genes. The amplicons were digested and amplified using the Ion AmpliSeq ${ }^{\mathrm{TM}}$ Library kit 2.0 (Life Technologies), according to the manufacturer's instructions. Finally, the template was loaded on an Ion $316^{\mathrm{TM}}$ chip and sequenced on a $\mathrm{PGM}^{\mathrm{TM}}$ sequencer with the Ion $\mathrm{PGM}^{\mathrm{TM}}$ sequencing $200 \mathrm{kit}$ v2 (Life Technologies), according to the manufacturer's protocols. Only mutations reported in the Human Gene Mutation Database (HGMD) at: http://www.hgmd.cf.ac.uk/ac/index.php and in the Catalogue Of Somatic Mutations In Cancer (COSMIC) at: http://www.sanger.ac.uk/genetics/CGP/cosmic/ were taken into account; silent or intronic mutations were not considered. All significant gene mutations identified on the Ion AmpliSeq Cancer Hotspot Panel were confirmed by Sanger sequencing of gene-specific amplicons (primer pairs provided on request). Briefly, polymerase chain reaction (PCR) was performed on $20 \mathrm{ng}$ of genomic DNA in a Veriti ${ }^{\circledR}$ 96-Well Fast Thermal Cycler (Life Technologies-ThermoFisher Scientific). All PCR-amplified products were directly sequenced using an automated fluorescence-cycle sequencer (ABI3130; Life Technologies-ThermoFisher Scientific). Sequencing analysis was conducted in duplicate and in both directions (forward and reverse) for all evaluated samples.

Morphology and phenotypic analysis of melanoma cells. Cells were grown at $80 \%$ confluence, fixed in $4 \%$ PFA and stained with $0,15 \%$ crystal violet. Cell images were captured using an 
inverted microscope (Leica, DMI6000). Cells were stained following incubation for $30 \mathrm{~min}$ at $+4^{\circ} \mathrm{C}$ with the following antibodies ( $5 \mu \mathrm{l} / \mathrm{ml})$ : mouse anti-human CD90 PE-Cy5, mesenchymal marker (BD Biosciences, Franklin Lakes, NJ, USA), human E-cadherin (CD324) APC, human CD133/2 (293C3)-PE (both from Miltenyi Biotec, GmbH, Bergisch Gladbach, Germany), mouse anti-human CD20 FITC and mouse antihuman CD44 FITC (both from BD Biosciences). After being washed with PBS, the samples were analyzed by FACS. The fold-change of the mean fluorescence intensity (MFI) values was used to show the differences in surface marker expression between drug-sensitive and -resistant melanoma cells. It was calculated from 3 independent experiments.

TGF- $\beta 1$ treatment. In order to induce EMT, all 3 melanoma cell lines (A375, 624.38 and 397) were treated with $2 \mathrm{ng} / \mathrm{ml}$ TGF- $\beta 1$ (Abcam, Milan, Italy) for $48 \mathrm{~h}$ and used for further investigations.

Quantitative real-time polymerase chain reaction ( $q R T-P C R$ ) analysis. Total RNA (300 ng) from the melanoma cell lines was converted to cDNA using M-MLV reverse transcriptase (Invitrogen, Life Technologies, Monza, MB, Italy). Primers used for E-cadherin were: forward, 5'-ACCACCTCCACAG CCACCGT-3' and reverse, 5'-GTCCAGTTGGCACTCGC CCC-3'; $\beta$-actin was used as an internal control (forward 5'-TTCTACAATGAGCTGCGTGTG-3' and reverse 5'-GGG GTGTTGAAGGTCTCAAA-3'). Experiments were performed in triplicate. qRT-PCR was carried out on an ABI PRISM 7900HT sequence detection system), using PowerUp SYBR-Green Master Mix (both from Applied Biosystems, Life Technologies, Grand Island, CA, USA).

Wound healing assay. Cells were seeded to confluency in 6 -well plates, and a wound was made in the center of the cell monolayer using a sterile $200-\mu 1$ pipette tip. The monolayer was rinsed 3 times with PBS and placed in RPMI-1640 medium. Phase contrast images were captured after $24 \mathrm{~h}$ and a digital image of the scar was captured at a magnification of x10 (Leica, DMI6000). All experiments were performed in triplicates.

Immunofluorescence assay. Cells were grown in RPMI on glass coverslips $(22 \times 22 \mathrm{~mm})$ and after $24 \mathrm{~h}$, fixed in $4 \%$ PFA and permeabilized in PBS containing $0.1 \%$ of Triton X-100. After blocking with BSA 5\% in PBS for $20 \mathrm{~min}$ at RT, cells were incubated with the following primary antibodies: vimentin (ab8069; 1:150; Abcam) and Oct4 (Oct4 C-10 antibody; 1:500; Santa Cruz Biotechnology, Inc., TX, USA) at $4^{\circ} \mathrm{C}$ overnight. After extensive washing, fluorescent secondary antibodies goat anti-rabbit (ab96885; Abcam), goat anti-mouse FITC (ImmunoReagents, Inc., Raleigh, NC, USA), diluted 1/500 in PBS were added for $90 \mathrm{~min}$ at $+4^{\circ} \mathrm{C}$. The cells were then counterstained with 4',6-diamidino-2-phenylindole (DAPI) (1:100 in PBS) for $7 \mathrm{~min}$ at $+4^{\circ} \mathrm{C}$, to stain the nuclei. The coverslips were washed again and mounted on slides using fluorescence mounting medium (Dako Diagnostics, Mississauga, ON, USA). Fluorescent images were captured using a Nikon upright microscope. The same immunofluorescence protocol was used for 3D human tissues (14), which were successively stained with hematoxylin and eosin (H\&E). Briefly, 3D human tissues from a differentiated and fullthickness skin reconstruction model of A375 melanoma cells were purchased from MatTek (Ashland, MA, USA). The 3D tissues were fed through the basolateral (bottom) surface, and incubated in duplicate with serum-free medium containing $0.2 \%$ dimethyl sulfoxide (DMSO) as a control or dabrafenib plus trametinib. The medium was replenished every other day, and the 3D tissues were collected on days 0,9 and 12 from the beginning of treatment and fixed with $4 \%$ PFA. Tissues were paraffin-embedded, serial-sectioned, deparaffinized in xylene and rehydrated through graded decreasing concentrations of alcohol and used for immunofluorescence studies and H\&E staining.

Protein extracts. Cells were lysed in lysis buffer [1\% Nonidet P-40, $150 \mathrm{mmol} / \mathrm{l} \mathrm{NaCl}, 10 \mathrm{mmol} / \mathrm{l}$ Tris ( $\mathrm{pH} \mathrm{7.4),} 1 \mathrm{mmol} / \mathrm{l}$ EDTA, $1 \mathrm{mmol} / 1$ EGTA (pH 8.0), $0.2 \mathrm{mmol} / 1$ sodium orthovanadate, $1 \mathrm{X}$ inhibitor cocktail) (Sigma-Aldrich, St. Louis, MO, USA) for $30 \mathrm{~min}$ at $4^{\circ} \mathrm{C}$ with constant agitation. Insoluble material was removed by centrifugation $\left(16,000 \mathrm{x} g\right.$ at $\left.4^{\circ} \mathrm{C}\right)$ for $15 \mathrm{~min}$ and the total protein concentration was determined in the supernatant by Bradford assay.

Western blot analysis. Western blotting was performed according to standard procedures. Rabbit monoclonal antibodies against Snail (diluted 1:1,000), rabbit polyclonal against Twist1 (diluted 1:1,000), and $\beta$-actin (diluted 1:1,000) were used. All the antibodies were purchased from Cell Signaling Technology (Danvers, MA, USA). Detection was achieved by HRP-conjugated anti-rabbit (1:10,000; Cell Signaling Technology) antibodies. Immune complexes were visualized by an enhanced chemiluminescence system (ECL Advance ${ }^{\mathrm{TM}}$; Amersham Pharmacia Biotech, Piscataway, NJ, USA). $\beta$-actin was used as a loading control. The image analysis was performed using ImageJ software (http://rsbweb.nih.gov/ij/).

Statistical analysis. Values are displayed as the mean \pm SEM of measurements of at least 3 independently performed experiments to avoid possible variation of cell cultures. Student's t-test was employed, and $\mathrm{P}<0.05$ was considered to be statistically significant.

\section{Results}

Genesis of melanoma cells resistant to $B-R A F$ inhibition. In order to characterize the phenotype of melanoma cells resistant to $\mathrm{B}-\mathrm{RAF}^{\mathrm{V} 600 \mathrm{E}}$ inhibition, we generated melanoma cells resistant to dabrafenib (a B-RAFi) using 3 melanoma cell lines, A375, 397 and 624.38, all carrying a B-RAF ${ }^{\mathrm{V} 600 \mathrm{E}}$ mutation and sensitive to dabrafenib.

As shown in Fig. 1A, cells plated at a low density in the presence of $30 \mathrm{nM}$ dabrafenib were able to form only a few sizeable colonies, after 7 days, comparable to the ones observed in the untreated cells, as expected.

We selected and expanded the few cells able to proliferate and form colonies under drug treatment, by growing them in medium containing increasing concentrations of dabrafenib, as described in Materials and methods. The resulting dabrafenibresistant melanoma cells are indicated as A375_B-RAFi (R), 
A
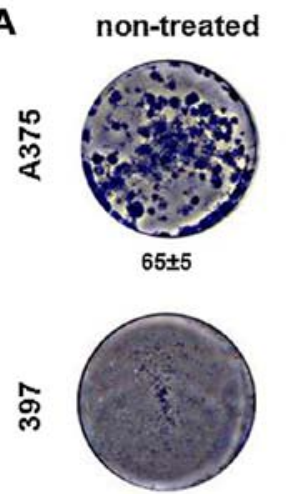

$50 \pm 4$

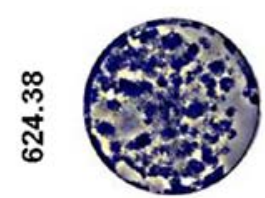

$78 \pm 7$

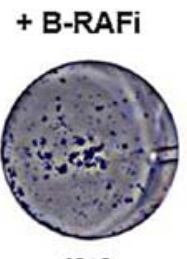

$19 \pm 3$

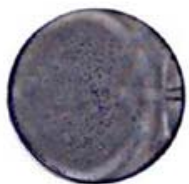

$15 \pm 2$

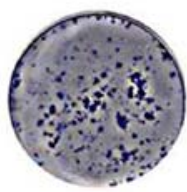

$45 \pm 5$ non-treated $\quad+$ B-RAFi
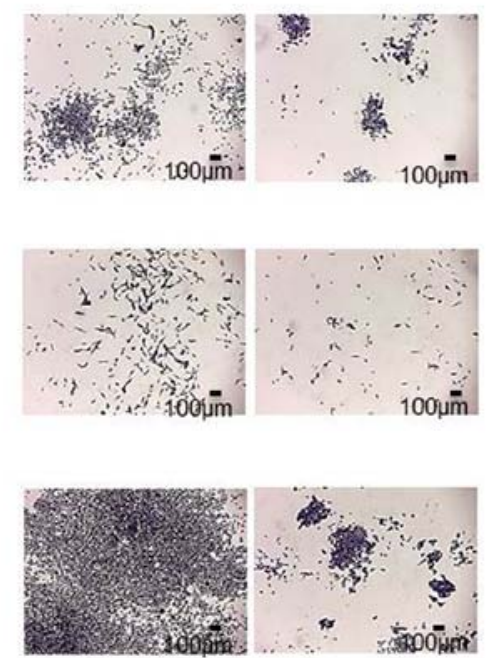


Figure 1. Melanoma resistance to the B-RAF inhibitor. Melanoma cell lines were treatment with the B-RAF inhibitor. A375, 397 and 624.38 melanoma cells were plated at clonal density and treated with dabrafenib $(30 \mathrm{nM})$. The medium and drug were replenished every 3 days, for 7 days. The cells were then fixed and stained with crystal violet. (A) Magnification of colonies is reported and the scale bar (100 $\mu \mathrm{m})$ is indicated. (B) Dose-response curves of melanoma cells A375 and A375_B-RAFi (R), 397 and 397_B-RAFi (R), and 624.38 and 624.38_B-RAFi (R) sensitive and resistant to B-RAF inhibition. The IC 50 values of B-RAFi were calculated using the logistic function (or sigmoidal) to fit dose-response curves, by GraphPad statistical software. The drug concentrations shown on the $\mathrm{x}$-axis are expressed in logarithmic form. The $\mathrm{y}$-axis indicates the percentage of cell death. The $\mathrm{IC}_{50}$ values are reported.

A
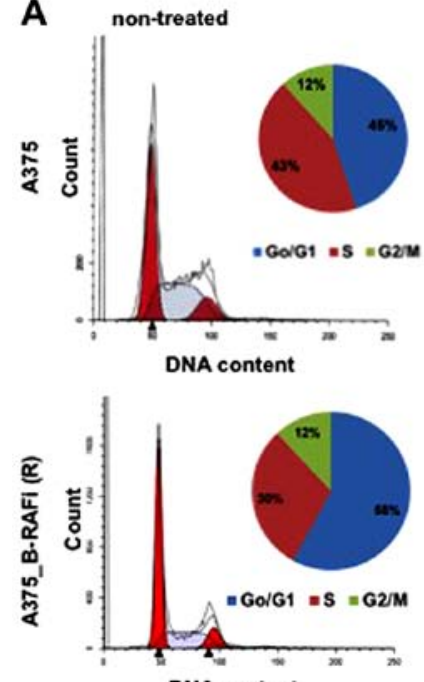

DNA content
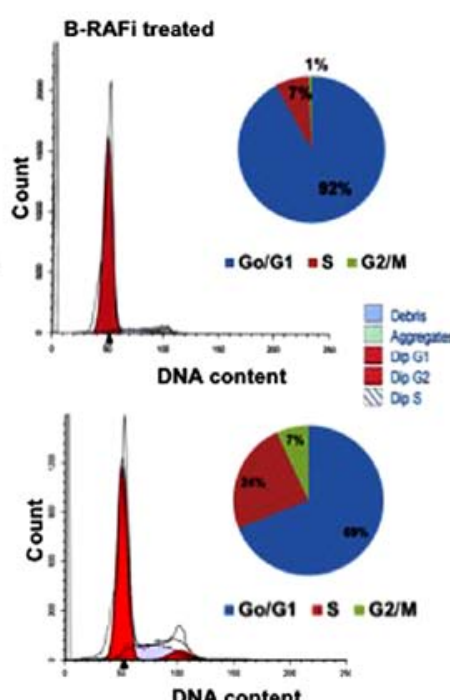

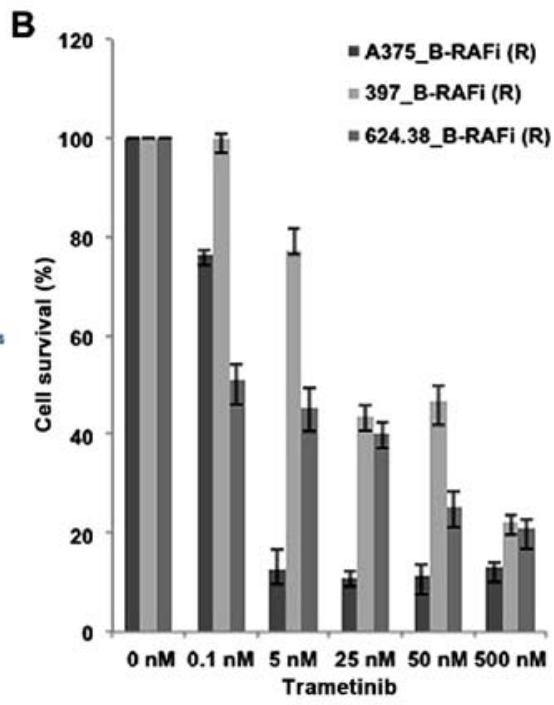

Figure 2. Characterization of melanoma cells resistant to the B-RAF inhibitor. Cell cycle profiles of melanoma cells sensitive and resistant to B-RAF inhibition. (A) Cell cycle progression analysis of A375 and A375_B-RAFi (R) melanoma cells after treatment with the B-RAF inhibitor for $72 \mathrm{~h}$, by staining fixed cells with propidium iodide (PI) to assess the percentage of cells in the G0/G1 (2N DNA content), G2/M (4N DNA content) and S phases (2-4N DNA content). The percentage of cells of each phase was calculated by flow cytometry and quantitated using ModFitLT V3 software. The pie charts show the mean \pm SD of the cell cycle distribution from 3 independent experiments. (B) All the dabrafenib-resistant melanoma cells were treated with different concentrations of trametinib, a Mek inhibitor, and the percentage of cell survival was examined. The results are expressed as the mean (percentage values) of 3 independent experiments performed in triplicate. The error bars represent $95 \%$ confidence intervals. 
397_B-RAFi (R) and 624.38_B-RAFi (R). Since the resistance to $\mathrm{B}-\mathrm{RAF}^{\mathrm{V} 600 \mathrm{E}}$ inhibition in melanoma is reversible and adaptive (15), the resistant cells were characterized, after their expansion, under drug selective pressure.

In order to confirm that A375_B-RAFi (R), 397_B-RAFi (R) and 624.38_B-RAFi (R) cells were resistant to dabrafenib treatment, the $\mathrm{IC}_{50}$ value was assessed, using the survival colorimetric assay. The A375 and the derived dabrafenib-resistant melanoma cells, A375_B-RAFi (R), were treated at increasing concentrations of B-RAFi $(0,0.1,1,5,25$ and $100 \mathrm{nM})$ for 6 days. Dose-response analysis revealed that dabrafenib-resistant cells showed an $\mathrm{IC}_{50}$ value of $110.5 \mu \mathrm{M}$ compared to the $9.5 \mathrm{nM}$ value determined for the dabrafenib-sensitive A375 counterparts (Fig. 1B). Similarly, a dabrafenib $\mathrm{IC}_{50}$ value of $280 \mu \mathrm{M}$ was determined for the 397_B-RAFi (R) cells, while a $0.006 \mathrm{nM}$ value was assessed for the 397 drug-sensitive cells (Fig. 1B). Finally, 624.38 and 624.38_B-RAFi (R) cells were treated at increasing concentrations of B-RAFi $(0,5,25,50,100$ and $500 \mathrm{nM}$ ) for 6 days. A dabrafenib $\mathrm{IC}_{50}$ value of $0.1 \mu \mathrm{M}$ was determined for the 624.38_B-RAFi (R) cells, while a $12 \mathrm{nM}$ value was assessed for the 624.38 drug-sensitive cells (Fig. 1B).

The effect on dabrafenib on the cell cycle profile was also examined by FACS analysis. Cell cycle profiles with PI revealed a marked difference between sensitive and resistant melanoma cells to dabrafenib. A significant accumulation of cells in the G0/G1 phase ( 91.9 vs. $45.1 \%$, dabrafenib-treated vs. untreated, respectively) with a concomitant decrease in the number of cells in the G2/M phase ( 0.69 vs. $11.8 \%$, dabrafenibtreated vs. untreated, respectively) was detected in the A375 cells when treated with the B-RAF inhibitor. Conversely, no significant $\mathrm{G} 0 / \mathrm{G} 1$ arrest in response to dabrafenib in the A375_B-RAFi (R) cells was observed (Fig. 2A). Similarly, an accumulation of cells in the G0/G1 phase with a decreased number of cells in the G2/M phase was observed for both 397 and 624.38 melanoma cells, when treated with the B-RAFi, while no significant cell cycle profile difference was detected for the resistant 397_B-RAFi (R) and 624.38_B-RAFi (R) melanoma cell lines, upon B-RAF inhibition (data not shown).

Finally, in order to ascertain whether the resistance to dabrafenib in these melanoma cells was associated with the reactivation of the MAPK signaling pathway (16), we assessed whether the treatment of these cells with MEK inhibitors compromised the viability of dabrafenib-resistant cells. As shown in Fig. 2B, these cells were sensitive to trametinib, a MEK inhibitor, supporting the association of resistance to dabrafenib with the reactivation of MAPK signaling.

Mutation analysis of melanoma cell lines. To assess the occurrence of discrepancies in mutation patterns during the development of the resistance status, dabrafenib-sensitive and -resistant melanoma cell lines were analyzed using the high throughput Ion Torrent sequencing technology, as described in Materials and methods. For the mutational screening the Ion AmpliSeq Cancer Hotspot Panel v2, containing $>200$ primer pairs producing short (average of $\sim 150 \mathrm{bp}$ ) amplicons which target hotspot regions of 50 main oncogenes and tumor-suppressor genes involved in tumorigenesis (Table I) was used.

All variants detected by NGS were confirmed through PCR-based Sanger sequencing. As shown in Table II, no difference in mutation patterns was observed between the sensitive and
Table I. Gene mutations identified on the Ion AmpliSeq Cancer Hotspot Panel and confirmed by Sanger sequencing of genespecific amplicons.

\begin{tabular}{|c|c|c|c|c|}
\hline$A B L 1$ & $E G F R$ & GNAS & KRAS & PTPN11 \\
\hline$A K T 1$ & $E R B B 2$ & $G N A Q$ & $M E T$ & $R B 1$ \\
\hline$A L K$ & $E R B B 4$ & HNF1A & $M L H 1$ & RET \\
\hline$A P C$ & EZH2 & $H R A S$ & $M P L$ & SMAD4 \\
\hline ATM & $F B X W 7$ & IDHI & NOTCHI & SMARCBI \\
\hline$B R A F$ & $F G F R I$ & $J A K 2$ & NPMI & $S M O$ \\
\hline $\mathrm{CDH1}$ & $F G F R 2$ & $J A K 3$ & NRAS & $S R C$ \\
\hline$C D K N 2 A$ & FGFR3 & $I D H 2$ & PDGFRA & STK11 \\
\hline CSF1R & FLT3 & $K D R$ & PIKЗCA & TP53 \\
\hline$C T N N B 1$ & GNA11 & KIT & PTEN & $V H L$ \\
\hline
\end{tabular}

resistant cell lines, strongly suggesting that development of resistance was independent of the acquisition of genetic alterations in the 50 most common oncogenes and tumor-suppressor genes examined (Table I). Moreover, mutations in the $C D K N 2 A$ and TP53 genes represented the main sequence variants associated with the $\mathrm{BRAF}^{\mathrm{V} 600 \mathrm{E}}$ mutation in our cell line series (Table II), further confirming the role of such crucial genes in controlling cell proliferation and survival in melanoma genesis.

Morphology and phenotype of melanoma cells resistant to $B-R A F$ inhibitor. In order to characterize the dabrafenib-resistant melanoma cells, their morphology and phenotype were examined. All cells were grown at 70-80\% confluence, stained with crystal violet and imaged using a light microscope, as described in Materials and methods.

All dabrafenib-resistant cells showed a morphological change compared to the A375, 397 and 624.38 cells, respectively (Fig. 3A). A bigger difference in the inter-cellular space was found between 624.38_B-RAFi (R) and 624.38 cells, while it was not highly evident between the other examined cell lines. These data revealed that melanoma resistance to dabrafenib was also associated with cell morphological changes.

Since it has been reported that EMT-like cancer cells exhibit the ability to survive chemotherapy and radiation therapy $(17,18)$, we examined the expression of mesenchymal CD90 (19) and E-cadherin epithelial markers in the dabrafenibresistant melanoma cells, and compared the obtained values with the ones determined for the dabrafenib-sensitive cells, by flow cytometry, as described in Materials and methods.

The expression level of E-cadherin was decreased in the A375_B-RAFi (R) and 397_B-RAFi (R) dabrafenib-resistant melanoma cells compared to the A375 and 397 dabrafenib-sensitive counterparts by 2 .1- and 2.2-fold, respectively, while it was slightly decreased in the 624.38_B-RAFi (R) cells compared to the 624.38 cells by $\sim 1.6$-fold. On the contrary, the CD90 marker expression level was increased by 2 .7- 1.4- and 2.3-fold in the A375_B-RAFi (R) vs. A375, 397_B-RAFi (R) vs. 397, and 624.38_B-RAFi (R) vs. 624.38 cells, respectively (Fig. 3B).

We conclude that melanoma cells, under dabrafenib exposure, exhibited morphological changes with a more emphasized mesenchymal phenotype. 
Table II. Mutations in the melanoma cell lines.

\begin{tabular}{|c|c|c|c|c|c|c|}
\hline Gene & Location & Function & Codon & Exon & Protein & Coding \\
\hline \multicolumn{7}{|c|}{ A375 and A375_B-RAFi (R) } \\
\hline BRAF & Exonic & Missense & GAG & 15 & p.Val600Glu & c.1799T $>\mathrm{A}$ \\
\hline CDKN2A & Exonic & Non-sense & TAG & 2 & p.Glu61Ter & c. $181 \mathrm{G}>\mathrm{T}$ \\
\hline CDKN2A & Exonic & Non-sense & TAG & 2 & p.Glu69Ter & c. $205 \mathrm{G}>\mathrm{T}$ \\
\hline TP53 & Exonic & Missense & CGC & 4 & p.Pro72Arg & c. $215 \mathrm{C}>\mathrm{G}$ \\
\hline \multicolumn{7}{|c|}{397 and 397_B-RAFi (R) } \\
\hline BRAF & Exonic & Missense & GAG & 15 & p.Val600Glu & c.1799T $>\mathrm{A}$ \\
\hline CDKN2A & Exonic & Homozygous deletion & $307 \mathrm{bp}$ & 2 & p.? (loss) & c.151_457del307 \\
\hline CTNNB1 & Exonic & Missense & TAT & 3 & p.Ser45Tyr & c. $134 \mathrm{C}>\mathrm{A}$ \\
\hline KDR & Exonic & Missense & CAT & 11 & p.Gln472His & c. $1416 \mathrm{~A}>\mathrm{T}$ \\
\hline NOTCH1 & Exonic & Non-frameshift deletion & GTG & 26 & p.Val1578del & c.4732_4734delGTG \\
\hline TP53 & Exonic & Missense & CGC & 4 & p.Pro72Arg & c. $215 \mathrm{C}>\mathrm{G}$ \\
\hline \multicolumn{7}{|c|}{624.38 and 624.38 B-RAFi (R) } \\
\hline BRAF & Exonic & Missense & GAG & 15 & p.Val600Glu & c.1799T>A \\
\hline KDR & Exonic & Missense & CAT & 11 & p.Gln472His & c. $1416 \mathrm{~A}>\mathrm{T}$ \\
\hline SMARCB1 & Exonic & Missense & AAA & 2 & p.Thr72Lys & c. $215 \mathrm{C}>\mathrm{A}$ \\
\hline TP53 & Exonic & Missense & CGC & 4 & p.Pro72Arg & c. $215 \mathrm{C}>\mathrm{G}$ \\
\hline TP53 & Exonic & Missense & TGG & 8 & p.Cys275Trp & c. $825 \mathrm{~T}>\mathrm{G}$ \\
\hline
\end{tabular}

A
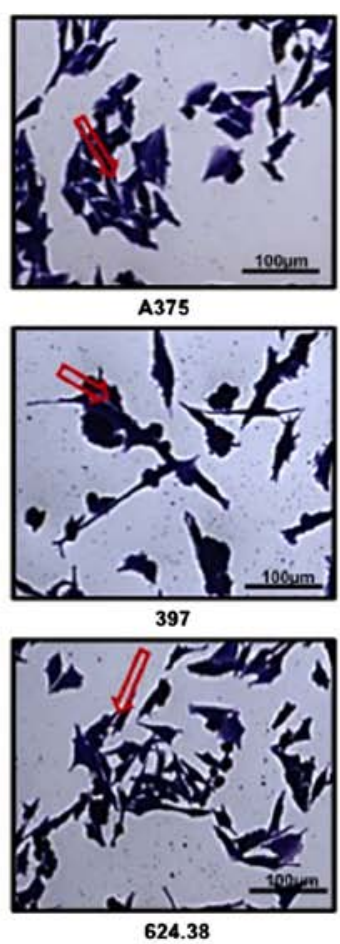

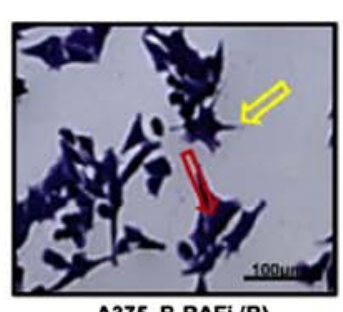

A375_B-RAFi (R)
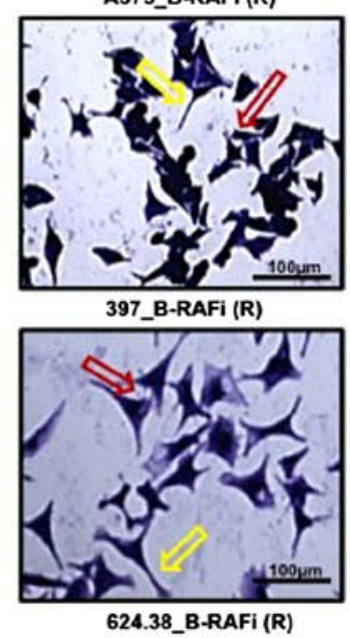

B

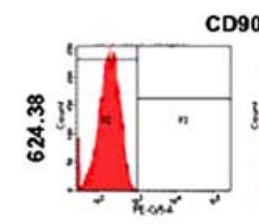

0
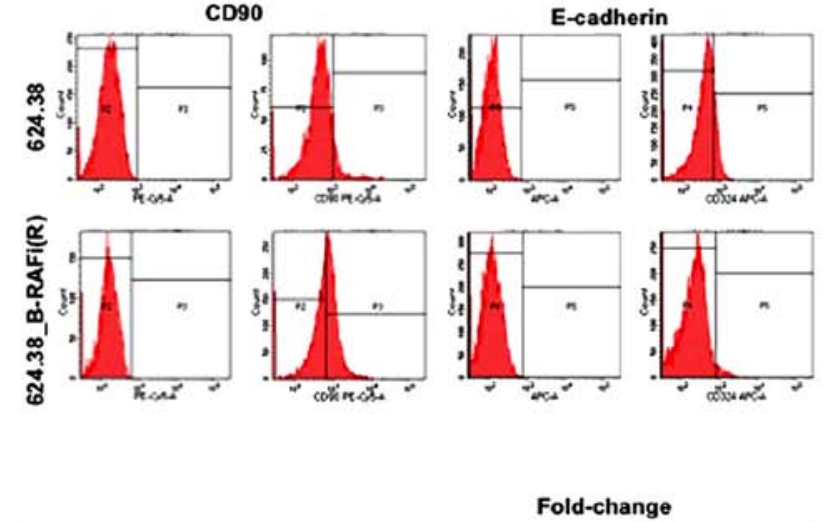

A375_B-RAFi (R) vs A375

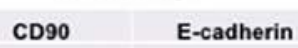

397_B-RAFi (R) vs 397

624.38_B-RAFi (R) vs 624

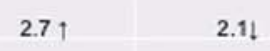

$1.4 \uparrow$

2.2!

$2.3 \uparrow$

$1.6 !$

Figure 3. Morphology of melanoma cells resistant to B-RAF and their mesenchymal and epithelial marker expression. (A) Morphology of melanoma cells. Representative images of all melanoma cells in a 2D culture system. Cells were stained with crystal violet. Pseudopodia and intracellular space are indicated with yellow and red arrows, respectively. The scale bar $(100 \mu \mathrm{m})$ is shown. (B) Mesenchymal and epithelial marker expression by FACS analysis. Representative images of CD90 and E-cadherin expression by FACS analysis in the 624.38 and 624.38_B-RAFi (R) melanoma cells and a summarized table of CD90 and E-cadherin expression analysis in all dabrafenib-resistant vs. -sensitive melanoma cells. The values in the table are expressed as the mean fluorescence intensity (MFI) fold-changes compared from 3 independent experiments to drug-sensitive melanoma cells.

Epithelial-to-mesenchymal-like phenotypic transition in melanoma cells resistant to dabrafenib. Although melanoma cells originate from melanocytes, not epithelial cells but neuroendocrine cells originating from the neural crest, their 
A
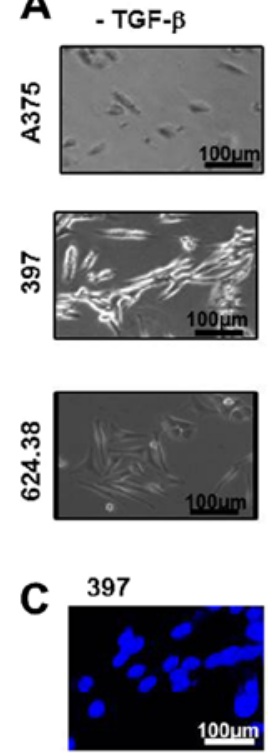

DAPI

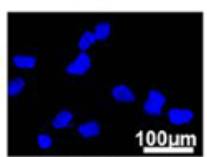

397_B-RAFi (R)

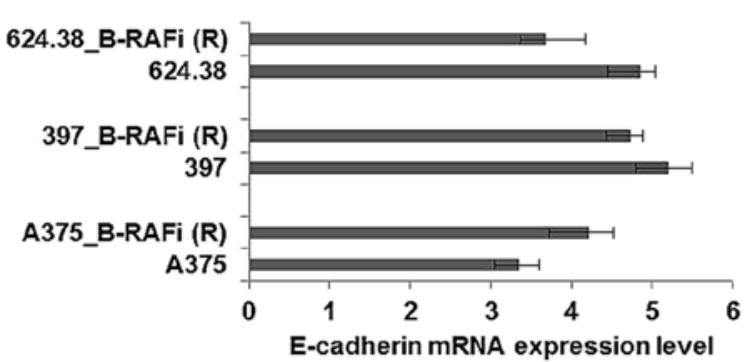

+ TGF- $\beta$
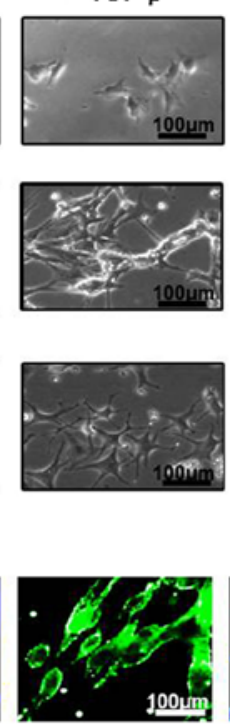

E-cadherin

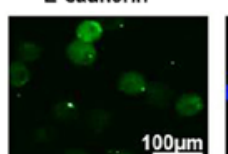

$100 \mathrm{um}$

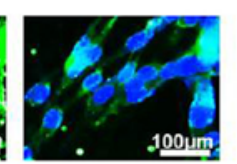

Merge

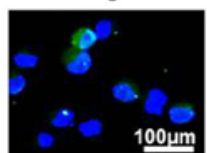

$100 \mu \mathrm{m}$



D
B
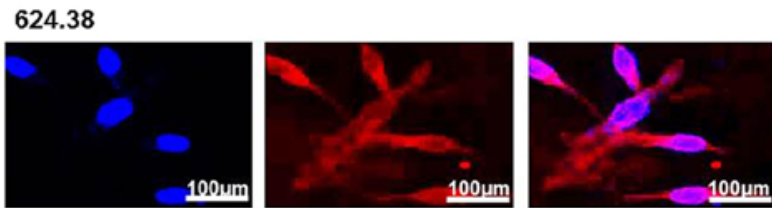

DAP

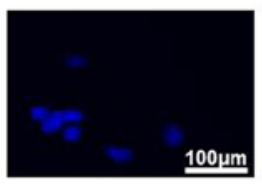

624.38_B-RAFi(R)

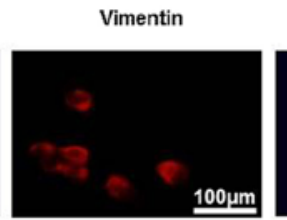

Merge

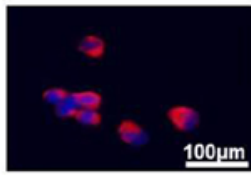

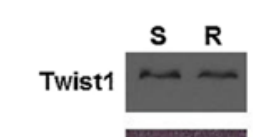
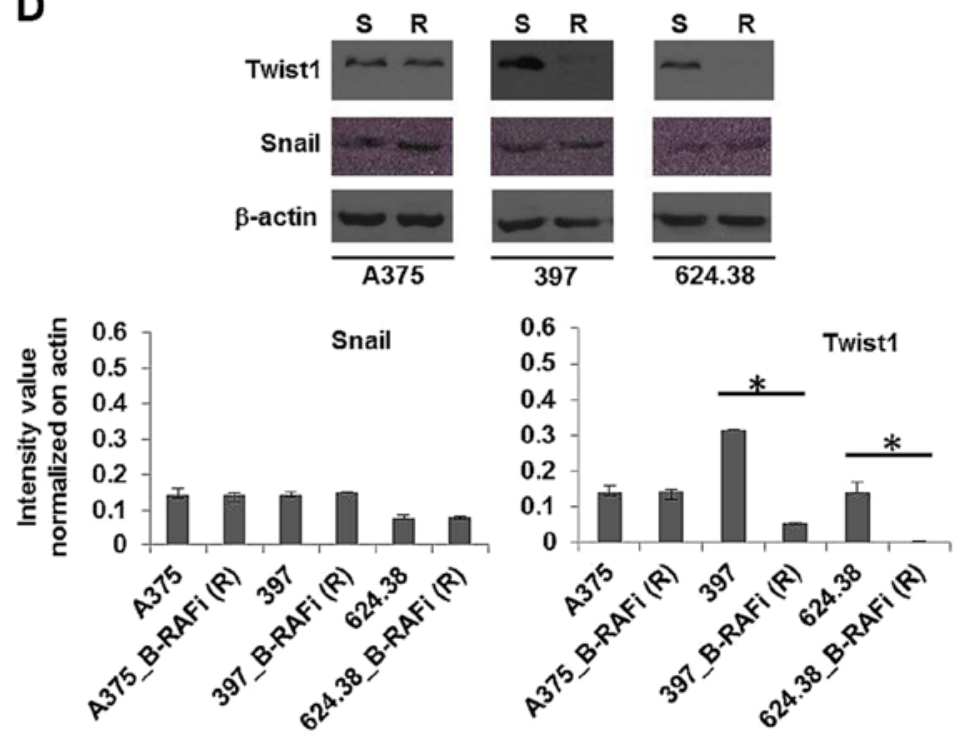

Figure 4. Phenotype of melanoma cells resistant to B-RAF inhibition. TGF- $\beta 1$-induced EMT in melanoma cells and their resistance to dabrafenib. All melanoma cells before and after TGF- $\beta 1$ treatment were observed using a phase-contrast microscope (magnification, $\mathrm{x} 10$ ). (A) The cell survival percentage of melanoma cells was examined, following dabrafenib exposure to the TGF- $\beta 1$-induced EMT and non-TGF- $\beta 1$-induced EMT melanoma cells. Results represent the means (percentage values) of 3 independent experiments performed in triplicate. The error bars show $95 \%$ confidence intervals. (B) Localization of the mesenchymal marker vimentin by immunofluorescence. Representative immunofluorescence staining of vimentin (red) in 624.38 and 624.38_BRAFi (R) melanoma cell lines. Melanoma 624.38 cells displayed predominantly cytoplasmic localization of vimentin while 624.38_B-RAFi (R) cells exhibited mainly peri-nuclear vimentin localization. (C) DAPI counterstain (blue) indicated the location of nuclei (magnification, $\mathrm{x} 20$ ). E-cadherin marker expression and localization by immunofluorescence. The mRNA expression level of the E-cadherin gene, by RT-PCR and representative immunofluorescence staining of E-cadherin (green) in 397 and 397_B-RAFi (R) melanoma cell lines. Melanoma 397 cells displayed predominantly E-cadherin localization on the membrane surface while in the 397_B-RAFi (R) cells it was detected in the cytoplasm. (D) Western blot analysis and quantification of Snail and Twist1 in all melanoma cell lines; ${ }^{*} \mathrm{P} \leq 0.005$.

ability to switch phenotype from proliferative to more invasive states, in a process similar to classical epithelial-mesenchymal transition (EMT), has been reported (20-22). In order to investigate whether the EMT was associated with the resistance to dabrafenib, EMT was induced in dabrafenib-sensitive melanoma cells by stimulation with TGF- $\beta 1$ (23), as described in Materials and methods, and then the cells were treated with dabrafenib (5 $\mathrm{nM})$.

As shown in Fig. 4A after treatment with TGF- $\beta 1$ for $48 \mathrm{~h}$ the cells underwent EMT and they were more sensitive to dabrafenib compared to the non-TGF- $\beta 1$-induced melanoma cells, suggesting that the observed drug resistance was not linked to the 'classical' TGF- $\beta 1$-induced EMT.

In order to shed more light on the phenotypic features of dabrafenib-resistant melanoma cells, we examined the network reorganization of vimentin filaments by immunofluorescence. We found that vimentin was mainly detected in the cytoplasm of the dabrafenib-sensitive melanoma cells, while it was observed in the peri-nuclear region in the dabrafenib-resistant cells (Fig. 4B), as expected in EMT.

Since downregulation of E-cadherin is regarded as a bona fide classical EMT inducer, we examined the E-cadherin transcriptional level in the dabrafenib-sensitive and -resistant melanoma cells, by RT-PCR, as described in Materials and methods. We observed that the E-cadherin level was unvaried between the dabrafenib-resistant and -sensitive cells, suggesting that EMT was not associated with the resistance to dabrafenib. However, a change in the E-cadherin protein distribution was observed between dabrafenib-resistant and -sensitive melanoma cells. In particular, E-cadherin was 


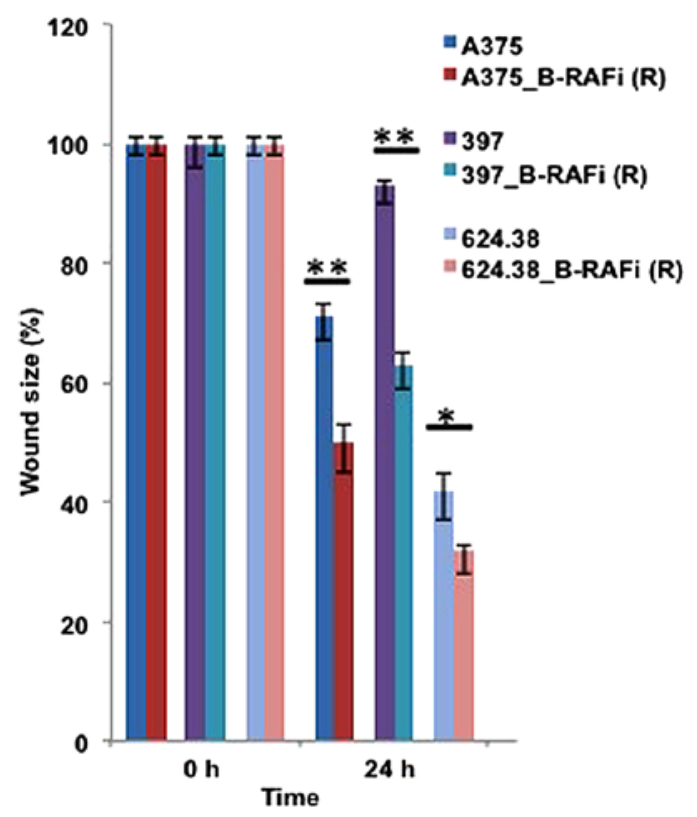

Figure 5. Analysis of cell motility. The different cell lines were grown at $80 \%$ confluence and a wound was created in the center of the cell monolayer. Wound size analyses performed at $24 \mathrm{~h}$ revealed enhanced motility in all 3 dabrafenib-resistant melanoma cell lines compared to the sensitive counterparts; ${ }^{*} \mathrm{P} \leq 0.005$ and ${ }^{* * *} \mathrm{P} \leq 0.001$, respectively.

A

\begin{tabular}{c|c|c|}
\hline \multicolumn{3}{|c|}{ Fold-change } \\
\hline$C D 20$ & $\mathrm{CD} 133$ & $\mathrm{CD} 44$ \\
\hline $2 \uparrow$ & $1.9 \uparrow$ & No Change \\
\hline $3.8 \uparrow$ & $2.6 \uparrow$ & $1.7 \uparrow$ \\
\hline $2 \uparrow$ & No Change & No Change \\
\hline
\end{tabular}

B
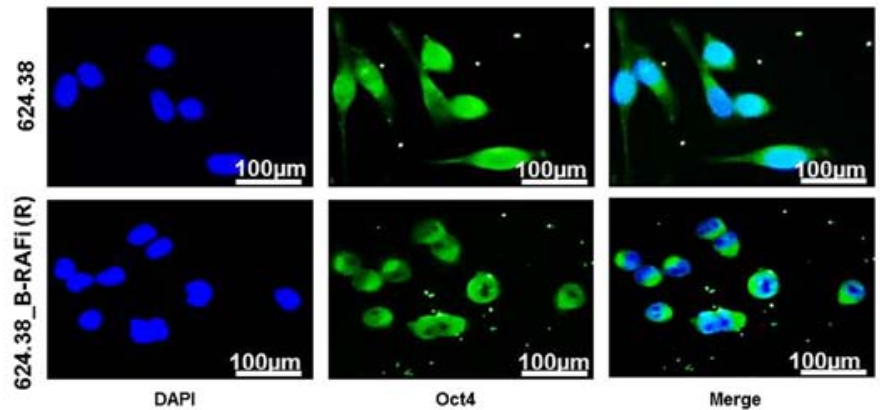

C

H\&E

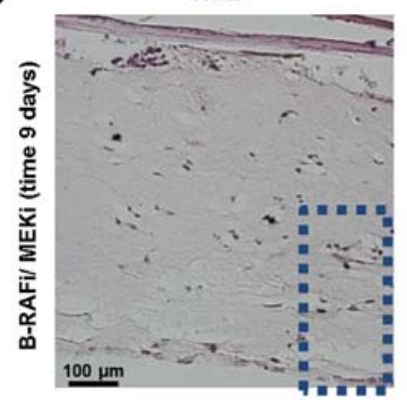

Oct4 (green) / Vimentin (red)


Figure 6. Stemness of melanoma cells resistant to B-RAF inhibition. CD20, CD133 and CD44 marker expression was determined by FACS analysis. (A) CD20, CD133 and CD44 marker expression in all melanoma cells resistant to dabrafenib vs. dabrafenib-sensitive cells are summarized in the table. Values in the table from 3 independent experiments are expressed as fold-changes in mean fluorescence intensity (MFI) compared to drug-sensitive cells. (B) Oct4 marker expression analysis by immunofluorescence. Immunofluorescence staining of Oct4 (green) in 624.38 and 624.38_B-RAFi (R) cells. Oct4 was mainly detected in the cytoplasm of the 624.38 cells, while it was found at peri-nuclear sites in resistant cells to B-RAFi. DAPI counterstain (blue) indicated the location of nuclei (magnification, x20). (C) Oct4 and vimentin marker expression analysis by immunofluorescence in a 3D-melanoma model. Immunofluorescence staining of Oct4 (green) and vimentin (red) of melanoma cell growth in human 3D tissues. H\&E staining showed a top bright red layer representing the epidermis; a successive layer of cells with dark blue nuclei consisting of the melanocyte layer and a bottom largely unstained layer representing the dermal stratum; representative sections from dabrafenib/trametinib drug-treated 3D tissues, stained with H\&E, Oct4 (green) and vimentin (red). A different magnification is shown of the dermal stratum. White arrows indicating Oct4 while orange arrows indicating vimentin are displayed. 
translocated from the membrane into the cytoplasm and nuclei of all drug-resistant cells (Fig. 4C), as expected in EMT.

Furthermore, all 3 melanoma cell lines resistant to dabrafenib exhibited unvaried expression levels of Snail, while Twist expression decreased in the 397_B-RAFi (R) vs. 397, and 624.38_B-RAFi (R) vs. 624.38 cells and was unvaried in the A375_B-RAFi (R) vs. A375 cells (Fig. 4D).

Collectively, these data revealed that melanoma cells, under drug exposure, underwent an epithelial-to-mesenchymal-like phenotypic transition, which was not a result of the activation of the TGF- $\beta 1$-induced EMT pathway.

Cell motility of melanoma cells resistant to the B-RAF inhibitor. Since cells undergoing EMT gain motility (24), we also investigated the cell motility of both dabrafenib-sensitive and -resistant melanoma cells, by wound healing assay, as described in Materials and methods.

As shown in Fig. 5, wound size analyses performed at $24 \mathrm{~h}$ revealed enhanced motility in the dabrafenib-resistant melanoma cells compared to their sensitive counterparts, further supporting an epithelial-to-mesenchymal-like phenotypic transition of melanoma cells under drug exposure.

Stem cell-like features of melanoma cells resistant to the $B-R A F$ inhibitor alone or combined with trametinib. It has been reported that EMT can produce a breast cancer stem cell (BCSC) phenotype in breast cancer, which explains the disease progression and relapse (25). In the present study, we investigated the expression of various cancer stem cell-like markers, such as CD20, CD133 and CD44 by FACS analysis and Oct4 expression in all melanoma cell lines by immunofluorescence.

We found 2 -fold increase in the expression level of CD20 in the A375_B-RAFi (R) and 624.38_B-RAFi (R) vs. the A375 and 624.38 cells, respectively, while it was more pronounced in the 397_B-RAFi (R) vs. the 397 cells with an increase in 3.8-fold (Fig. 6A). CD133 marker expression was increased in the A375_B-RAFi (R) and 397_B-RAFi (R) vs. the A375 and 397 cells, respectively, while it was unmodified in the 624.38_B-RAFi (R) vs. 624.38 cells. Finally, the expression level of CD44 was upregulated only in the 397_B-RAFi (R) vs. 397 cells, while it was unaltered in all the remaining cells (Fig. 6A).

Furthermore, we examined Oct4 expression by immunofluorescence, as described in Materials and methods. Oct4 is one among the regulatory core transcription factors involved in the maintenance of stemness in mesenchymal stem cells (26) as well as a factor induced by EMT (27). Oct4 protein was mainly detected at the cytoplasmic level in the melanoma cells sensitive to B-RAF inhibition, while it was located at the perinuclear site and in the nucleus of all 3 dabrafenib-resistant melanoma cell lines (Fig. 6B), suggesting Oct4 activation in all the resistant cancer cells $(28,29)$.

Vimentin and Oct4 localization in a 3-dimensional skin reconstruction model of A375 melanoma cells. In a 3-dimensional (3D) skin reconstruction model of A375 melanoma cells, we previously observed individual proliferating melanoma cells infiltrating the dermis under BRAFi/MEKi drug treatment (14), where MEKi used was trametinib drug. Vimentin and Oct4 expression was also analyzed in these
3D tissues by immunofluorescence. We observed that the melanoma cells infiltrating the dermis expressed vimentin and Oct4 in the peri-nuclear sites. Oct4 was also detected in the nuclei. These findings support the mesenchymal and stem-like phenotype of these cells (Fig. 6C).

\section{Discussion}

The advancement of knowledge in melanoma biology has led to the development of so-called 'targeted therapeutics', such as dabrafenib and trametinib which target B-RAF ${ }^{\mathrm{V} 600 \mathrm{E}}$ and the MEK protein, respectively. However, development of drug resistance due to the activation of alternative growthcontrolling pathways remains a critical issue for melanoma treatment with such compounds, although emerging concepts of computational modeling are making strides in predicting the response to therapy $(30,31)$.

In the present study, we observed that 3 different melanoma cell lines, all derived from MM patients and carrying the $\mathrm{B}-\mathrm{RAF}^{\mathrm{V} 600 \mathrm{E}}$ mutation, contained a few cells able to proliferate upon treatment with dabrafenib (B-RAFi), suggesting the existence of melanoma cells able to escape the drug effect and responsible for melanoma progression. We found that all dabrafenib-resistant cells had reactivated MAPK signaling, and exhibited a more elongated shape with increased pseudopodia formation and inter-cellular space as compared to the drugsensitive counterparts. Notably, these morphological changes were accompanied by an increased expression of CD90, a mesenchymal marker (19), decreased expression of E-cadherin, an epithelial marker, suggesting the mesenchymal features of the dabrafenib-resistant melanoma cells. This finding was further supported by the detection of vimentin at peri-nuclear sites in the resistant cells, while it was observed in the cytoplasm of melanoma cells sensitive to the drugs. Furthermore, all dabrafenib-resistant melanoma cells showed higher cell motility than the dabrafenib-sensitive ones, suggesting an epithelial-to-mesenchymal phenotypic transition of these cells. However, downregulation of E-cadherin, which is regarded as a bona fide classical EMT inducer, was not detected (25), suggesting that melanoma cells undergo an epithelial-to-mesenchymal-like transition or a pathological EMT. This finding was further supported from the observation that TGF- $\beta 1$-induced EMT was not linked to dabrafenib resistance.

Moreover, sequence analysis by Ion Torrent technology indicated a lack of discrepancies in mutational patterns of main cancer genes between the drug-sensitive and drugresistant melanoma cells, indicating that the genetic alterations in the examined oncogenes and tumor-suppressor genes were not involved in the phenotypic changes observed in the drug-resistant cells.

In the past decade, the developmental EMT process has been recognized to play pivotal and complex roles in promoting cancer invasion and metastasis as well as inducing drug resistance $(26,32,33)$. It has been reported that genes involved in the activation of EMT, such as Wnt, Oct4, EGF and Nanog, are expressed in cells exhibiting stem cell properties and that EMT plays a critical role in the cancer stem cell (CSC) generation in breast cancer $(26,34)$. We investigated the expression of Oct4, CD20, CD133 and CD44 stem cell markers in our series. Oct4 is a regulatory core transcription factor, involved in the 
maintenance of stemness in mesenchymal stem cells (26). CD20 has been associated with melanoma CSCs, while discordant data have been reported regarding CD133 as a melanoma cancer stem cell marker and CD44 has not been found to be associated with melanoma stem cells (35). We found different stem-cell marker traits between dabrafenib-resistant and -sensitive cells, which could reflect the context or microenvironment from which the cells were derived (32). Notably, the expression of Oct4 was observed in the peri-nuclear sites and in the nuclei of the resistant cells, while it was detected in the cytoplasm of the melanoma cells sensitive to drugs.

We conclude that melanoma resistance to dabrafenib was associated with MAPK signaling reactivation accompanied by an epithelial-to-mesenchymal-like phenotypic transition as well as with the acquirement of stem-like phenotypic features, which may help to explain the ability of the cells to continue to proliferate under drug conditions.

These data support the concept of pathological EMT as well as the concept linking the EMT process and the generation of CSCs. Understanding the molecular signaling associated with this EMT-like or pathological EMT has become critical for providing effective target drugs, toward successful therapeutic strategies in melanoma. Therefore, a more detailed molecular characterization of this cell-subset may be crucial to modulate such cellular programs for therapeutic purposes.

\section{Acknowledgements}

The authors would like to thank the technical support provided by the Integrated Microscopy facility at IGB and Dr Antonio Simeone for providing the Oct 4 antibody. The present study was supported by a grant from Medical Research in Italy (RBNE08HM7T-003) to E.J.P. and Regione Autonoma Sardegna to G.Pa. Special thanks to Fondazione Melanoma Onlus for providing financial support.

\section{References}

1. Carvajal RD, Marghoob AA, Kaushal A, Kehrer JD, Ko C and Brady MS: Melanoma and other skin cancers. Cancer Network. Home of the Journal Oncology 1: 1-23, 2014.

2. Tarver T: Cancer facts and figures 2012. American Cancer Society (ACS). J Consumer Health Internet 16: 366-367, 2012.

3. Candido S, Rapisarda V, Marconi A, Malaponte G, Bevelacqua V, Gangemi P, Scalisi A, McCubrey JA, Maestro R, Spandidos DA, et al: Analysis of the $B-R a f^{\mathrm{V} 600 \mathrm{E}}$ mutation in cutaneous melanoma patients with occupational sun exposure. Oncol Rep 31: 1079-1082, 2014.

4. Falzone L, Salemi R, Travali S, Scalisi A, McCubrey JA, Candido S and Libra M: MMP-9 overexpression is associated with intragenic hypermethylation of MMP9 gene in melanoma. Aging 8: 933-944, 2016.

5. Bernardo-Faura M, Massen S, Falk CS, Brady NR and Eils R: Data-derived modeling characterizes plasticity of MAPK signaling in melanoma. PLOS Comput Biol 10: e1003795, 2014.

6. Ascierto PA, Grimaldi AM, Anderson AC, Bifulco C, Cochran A Garbe C, Eggermont AM, Faries M, Ferrone S, Gershenwald JE, et al: Future perspectives in melanoma research: Meeting report from the 'Melanoma Bridge', Napoli, December 5th-8th 2013. J Transl Med 12: 277, 2014.

7. Das Thakur M, Salangsang F, Landman AS, Sellers WR, Pryer NK, Levesque MP, Dummer R, McMahon M and Stuart DD: Modelling vemurafenib resistance in melanoma reveals a strategy to forestall drug resistance. Nature 494: $251-255,2013$.
8. Nazarian R, Shi H, Wang Q, Kong X, Koya RC, Lee H, Chen Z, Lee MK, Attar N, Sazegar H, et al: Melanomas acquire resistance to B-RAF(V600E) inhibition by RTK or N-RAS upregulation. Nature 468: 973-977, 2010.

9. Shi H, Moriceau G, Kong X, Lee MK, Lee H, Koya RC, Ng C, Chodon T,Scolyer RA,Dahlman KB, et al: Melanoma whole-exome sequencing identifies (V600E)B-RAF amplification-mediated acquired B-RAF inhibitor resistance. Nat Commun 3: 724, 2012.

10. Shi H, Hugo W, Kong X, Hong A, Koya RC, Moriceau G, Chodon T, Guo R, Johnson DB, Dahlman KB, et al: Acquired resistance and clonal evolution in melanoma during BRAF inhibitor therapy. Cancer Discov 4: 80-93, 2014.

11. Rizos H, Menzies AM, Pupo GM, Carlino MS, Fung C, Hyman J, Haydu LE, Mijatov B, Becker TM, Boyd SC, et al: BRAF inhibitor resistance mechanisms in metastatic melanoma: Spectrum and clinical impact. Clin Cancer Res 20: 1965-1977, 2014.

12. Van Allen EM, Wagle N, Sucker A, Treacy DJ, Johannessen CM, Goetz EM, Place CS, Taylor-Weiner A, Whittaker S, Kryukov GV, et al; Dermatologic Cooperative Oncology Group of Germany (DeCOG): The genetic landscape of clinical resistance to RAF inhibition in metastatic melanoma. Cancer Discov 4: 94-109, 2014.

13. Ascierto PA, Marincola FM and Atkins MB: What's new in melanoma? Combination! J Transl Med 13: 213, 2015.

14. Caputo E, Miceli R, Motti ML, Taté R, Fratangelo F, Botti G, Mozzillo N, Carriero MV, Cavalcanti E, Palmieri G, et al: AurkA inhibitors enhance the effects of B-RAF and MEK inhibitors in melanoma treatment. J Transl Med 12: 216, 2014.

15. Sun C, Wang L, Huang S, Heynen GJ, Prahallad A, Robert C, Haanen J, Blank C, Wesseling J, Willems SM, et al: Reversible and adaptive resistance to $\mathrm{BRAF}(\mathrm{V} 600 \mathrm{E})$ inhibition in melanoma. Nature 508: 118-122, 2014.

16. Sanchez-Laorden B, Viros A, Girotti MR, Pedersen M, Saturno G, Zambon A, Niculescu-Duvaz D, Turajlic S, Hayes A, Gore M, et al: BRAF inhibitors induce metastasis in RAS mutant or inhibitor-resistant melanoma cells by reactivating MEK and ERK signaling. Sci Signal 7: ra30, 2014

17. Jung JW, Hwang SY, Hwang JS, Oh ES, Park S and Han IO: Ionising radiation induces changes associated with epithelialmesenchymal transdifferentiation and increased cell motility of A549 lung epithelial cells. Eur J Cancer 43: 1214-1224, 2007.

18. Yang AD, Fan F, Camp ER, van Buren G, Liu W, Somcio R, Gray MJ, Cheng H, Hoff PM and Ellis LM: Chronic oxaliplatin resistance induces epithelial-to-mesenchymal transition in colorectal cancer cell lines. Clin Cancer Res 12: 4147-4153, 2006.

19. Dominici M, Le Blanc K, Mueller I, Slaper-Cortenbach I, Marini F, Krause D, Deans R, Keating A, Prockop Dj and Horwitz E: Minimal criteria for defining multipotent mesenchymal stromal cells. The International Society for Cellular Therapy position statement. Cytotherapy 8: 315-317, 2006.

20. Hoek KS, Eichhoff OM, Schlegel NC, Döbbeling U, Kobert N, Schaerer L, Hemmi S and Dummer R: In vivo switching of human melanoma cells between proliferative and invasive states. Cancer Res 68: 650-656, 2008.

21. Wehbe M, Soudja SM, Mas A, Chasson L, Guinamard R, de Tenbossche CP, Verdeil G, Van den Eynde B and Schmitt-Verhulst AM: Epithelial-mesenchymal-transition-like and TGF $\beta$ pathways associated with autochthonous inflammatory melanoma development in mice. PLoS One 7: e49419, 2012.

22. Jayachandran A, Anaka M, Prithviraj P, Hudson C, McKeown SJ, Lo PH, Vella LJ, Goding CR, Cebon J and Behren A: Thrombospondin 1 promotes an aggressive phenotype through epithelial-to-mesenchymal transition in human melanoma. Oncotarget 5: 5782-5797, 2014

23. Nawshad A, Lagamba D, Polad A and Hay ED: Transforming growth factor-beta signaling during epithelial-mesenchymal transformation: Implications for embryogenesis and tumor metastasis. Cells Tissues Organs 179: 11-23, 2005.

24. Gavert N and Ben-Ze'ev A: Epithelial-mesenchymal transition and the invasive potential of tumors. Trends Mol Med 14: 199-209, 2008.

25. Nieto MA: The ins and outs of the epithelial to mesenchymal transition in health and disease. Annu Rev Cell Dev Biol 27: 347-376, 2011.

26. Santisteban M, Reiman JM, Asiedu MK, Behrens MD, Nassar A, Kalli KR, Haluska P, Ingle JN, Hartmann LC, Manjili MH, et al: Immune-induced epithelial to mesenchymal transition in vivo generates breast cancer stem cells. Cancer Res 69: 2887-2895, 2009.

27. Zimmerer RM, Korn P, Demougin P, Kampmann A, Kokemüller H, Eckardt AM, Gellrich NC and Tavassol F: Functional features of cancer stem cells in melanoma cell lines. Cancer Cell Int 13: 78, 2013. 
28. Kashyap V, Rezende NC, Scotland KB, Shaffer SM, Persson JL, Gudas LJ and Mongan NP: Regulation of stem cell pluripotency and differentiation involves a mutual regulatory circuit of the NANOG, OCT4, and SOX2 pluripotency transcription factors with polycomb repressive complexes and stem cell microRNAs. Stem Cells Dev 18: 1093-1108, 2009.

29. Wen KM, Zhang GH, Li J, Chen ZQ, Cheng YL, Su X and Zeng QL: OCT4B1 promotes cell growth, migration and invasion suppressing sensitivity to oxaliplatin in colon cancer. Oncol Rep 34: 2943-2952, 2015

30. Pennisi M, Russo G, Di Salvatore V, Candido S, Libra M and Pappalardo F: Computational modeling in melanoma for novel drug discovery. Expert Opin Drug Discov 11: 609-621, 2016.

31. Pappalardo F, Russo G, Candido S, Pennisi M, Cavalieri S, Motta S, McCubrey JA, Nicoletti F and Libra M: Computational modeling of PI3K/AKT and MAPK signaling pathways in melanoma cancer. PLoS One 11: e0152104, 2016.
32. Bissell MJ and Labarge MA: Context, tissue plasticity, and cancer: Are tumor stem cells also regulated by the microenvironment? Cancer Cell 7: 17-23, 2005.

33. Yin X, Zhang BH, Zheng SS, Gao DM, Qiu SJ, Wu WZ and Ren ZG: Coexpression of gene Oct4 and Nanog initiates stem cell characteristics in hepatocellular carcinoma and promotes epithelial-mesenchymal transition through activation of Stat3/Snail signaling. J Hematol Oncol 8: 23, 2015.

34. Mani SA, Guo W, Liao MJ, Eaton EN, Ayyanan A, Zhou AY, Brooks M, Reinhard F, Zhang CC, Shipitsin M, et al: The epithelial-mesenchymal transition generates cells with properties of stem cells. Cell 133: 704-715, 2008

35. Medema JP: Cancer stem cells: The challenges ahead. Nat Cell Biol 15: 338-344, 2013. 